Copyright (C) 2002 IEEE. Reprinted from

IEEE Transactions on Instrumentation and Measurement, 2002; 51

(2):299-309

This material is posted here with permission of the IEEE. Such permission of the IEEE does not in any way imply IEEE endorsement of any of the University of Adelaide's products or services. Internal or personal use of this material is permitted. However, permission to reprint/republish this material for advertising or promotional purposes or for creating new collective works for resale or redistribution must be obtained from the IEEE by writing to pubs-permissions@ieee.org.

By choosing to view this document, you agree to all provisions of the copyright laws protecting it. 


\title{
A Review of Stochastic Resonance: Circuits and Measurement
}

\author{
Gregory P. Harmer, Bruce R. Davis, Member, IEEE, and Derek Abbott, Senior Member, IEEE
}

\begin{abstract}
Noise in dynamical systems is usually considered a nuisance. However, in certain nonlinear systems, including electronic circuits and biological sensory systems, the presence of noise can enhance the detection of weak signals. The phenomenon is termed stochastic resonance and is of great interest for electronic instrumentation.

We review and investigate the stochastic resonance of several bistable circuits. A new type of $S$ characteristic circuit is demonstrated using simple nonlinear elements with an operational amplifier. Using this circuit, the effects on stochastic resonance were determined as the slope of the $S$ shaped characteristic curve was varied.
\end{abstract}

Index Terms-Bistable circuits, level crossing circuits, stochastic resonance.

\section{INTRODUCTION}

$\mathbf{W}$ HEN noise is added to a system, the output usually deteriorates in quality. However, in some systems, adding the right amount of noise can enhance the system output or response. For these systems, it can be shown that there exists a nonzero value of noise that gives an optimal value for the signal-to-noise ratio (SNR) of the system.

Stochastic resonance (SR) is characterized by the response of a system to noise, the signal-to-noise ratio. The SNR rises sharply to a maximum value, then gradually decreases for higher noise intensities, where SR occurs, as noise is added to a system. It is seen from this description of SR that it is not strictly a resonance as the increased response is not due to a natural frequency of the system. The alternative use of the word resonance is derived from the SNR having a peak due to some other parameter-in this case, input noise amplitude.

The essential ingredient for SR is a nonlinear dynamical system, which typically has a periodic signal and noise at the input and an output that is a function of the inputs as well as the internal dynamics of the system. The nonlinear component of the dynamical system is sometimes provided by a threshold which must be crossed for the output to be changed or detected. A nonlinear system is essential for SR to exist, since in a system that is well characterized by linear response theory, the SNR at the output must be proportional to the SNR at the input. Thus, any increase in the input noise will result in a decrease in the output SNR, which is in contrast to the characteristic of SR.

Manuscript received April 21, 2000; revised February 10, 2002. This work was supported by the Australian Research Council and the Sir Ross and Sir K. Smith fund.

The authors are with the Department of Electrical and Electronic Engineering, University of Adelaide, Adelaide, SA, Australia.

G. P. Harmer and D. Abbott are also with the Centre for Biomedical Engineering (CBME) University of Adelaide, Adelaide, SA, Australia (e-mail: dabbott@eleceng.adelaide.edu.au).

Publisher Item Identifier S 0018-9456(02)04312-7.
Engineers have normally sought to minimize the effects of noise in electronic circuits and communication systems. Today, however, it is acknowledged that noise or random motion is beneficial in breaking up the quantization pattern in a video signal [1], in the dithering of analog to digital converters (ADCs) [2], in the area of Brownian ratchets [3], and in the physics of mixed powders [4]-[6]. Also, it is known that when training a neural network, adding noise to the training data set can improve network generalization, i.e., the neural network's ability to fit real data outside of the initial training set [7]. Noise even plays a role in game theory [8], [9], number theory [10], and cosmology [11]. Furthermore, recent research has established that noise can play a constructive role in the detection of weak periodic signals via a mechanism known as stochastic resonance. In essence, SR is a nonlinear cooperative effect in which a weak periodic stimulus influences large-scale fluctuations, the result being that the periodic component is greatly enhanced.

Stochastic resonance is now known to occur in a wide range of physical systems; however, it was originally proposed by Benzi [12] in 1980, as an explanation of the behavior of the Earth's ice ages, which exhibit a 100000 year periodicity. This was closely followed by a number of papers [13]-[15] describing a general dynamical mechanism whereby small periodic perturbations could be greatly amplified by large environmental fluctuations.

Experimentally, SR was first demonstrated with a noise driven electronic circuit based on the Schmitt trigger [16]. This work was also the first to characterize the phenomenon in terms of a signal-to-noise ratio. It took more than five years before the interest of physicists was sparked by the demonstration of SR in a bistable ring-laser experiment [17].

Stochastic resonance has been reported in a variety of physical systems [18]-[20] and the classical theory is well established [21]-[24]. The concept of SR has been extended to a class of systems for which the signal-to-noise ratio may display a multiplicity of maxima with increasing noise. This was shown for the first time in [25], where a response for a periodic force is enhanced, not only for an optimized amount of noise, but also at multiple values of the noise level. Now SR has crossed disciplinary boundaries: its role in sensory biology is being explored in experiments on single crayfish neurons and in perceptive brain function by experiments on people's ability to resolve ambiguous figures. SR has been shown to enhance the sensitivity of mechanoelectrical transduction in the ear of a frog [26] and it is likely to occur in the human ear [27] also. These new efforts, together with attempts to exploit SR for technological advantage, are the main trends in current research on this topic. There are even indications that SR may be useful in medical and environmental science. 
Three case studies are considered to show the diversity of disciplines that SR covers. Some theory is presented for noise and bistable systems. Initially, simulations are used to check for SR from electrical circuit characteristics. Then, we construct some circuits to demonstrate the measurement of SR and outline possible application areas.

\section{EXAMPLES OF SR}

In order to gain an appreciation of the concepts associated with stochastic resonance and the diversity of fields it covers, some case studies are reviewed. The first example examines the sensing systems of the crayfish [28]-[30], the second relates to visual perception [31]-[33], and the third to public opinion formation [34].

\section{A. Crayfish Sensory Systems}

The primary objective of the sense organs is to detect faint information bearing signals in noisy environments. Stochastic resonance plays an essential role in biological sensing systems and thus is likely to prove fruitful.

The system of the mechanoreceptor cells found on the tail of the ordinary crayfish, Procambarus clarkii, is described. These cells, which end in fine hairs, are specialized to detect weak (and probably periodic) water motions such as those generated by the waving tail of a nearby predatory fish. The hair cells most likely serve as an early warning system, which may explain why crayfish are one of the oldest and most well adapted animals around.

When moved, the hair generates a nerve impulse, measurable as a short duration electric spike. The impulse travels to a bundle of nerve cells, which handle all incoming information from the hair cells. These nerve cells make basic decisions such as activating the animal's escape reflexes. Evidence indicates that the sensory neurons function much like nonlinear threshold detectors. Below a certain level, very weak motion of the hair does not generate nerve impulses. This simplicity of the system makes it a useful one to study.

An experiment to mimic the noisy environment in which crayfish normally live using an excised piece of crayfish tail is described in [28]. The procedure essentially involved moving the tail periodically in water with an electromechanical transducer until the nerve impulses were nearly undetectable, then adding noise. The resulting signal-to-noise ratio, shown in Fig. 1, illustrates stochastic resonance.

Fig. 1 shows the results for theoretical predictions and an electronic simulation of a threshold detector for comparison. The discrepancies at high and low noise levels are due respectively to neurons exhibiting a refractory period and unavoidable internal noise in sensory systems.

\section{B. Visual Perception}

Stochastic resonance can be observed when dealing with visual perception of ambiguous figures [31], [33]. Perception of ambiguous figures, such as a wire frame cube, are characterized by noisy bistable dynamics. Two different interpretations of the cube are alternatively perceived by the observer with a

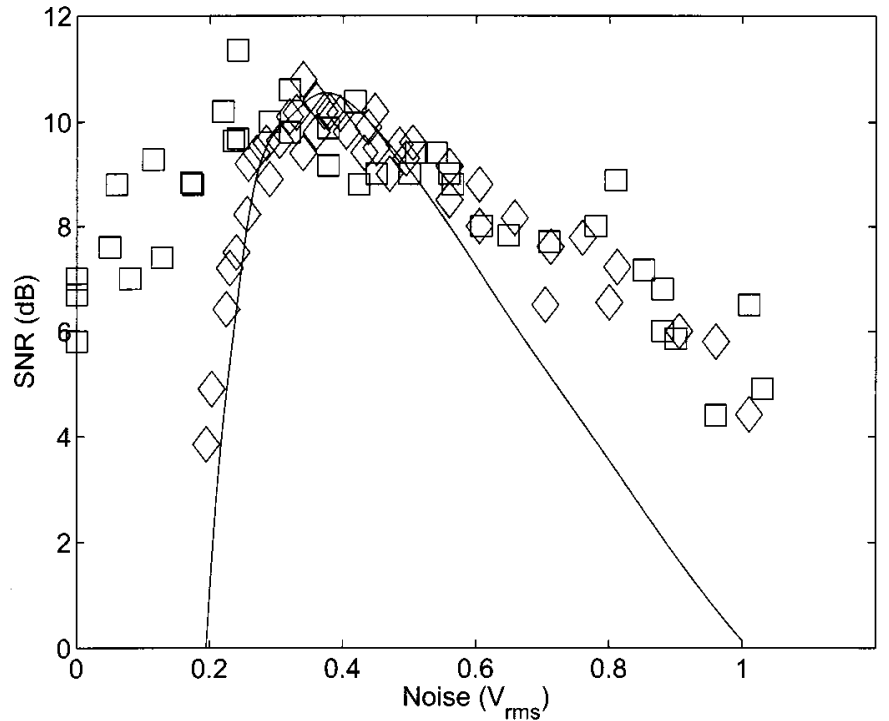

Fig. 1. SNR of the crayfish mechanoreceptors when external noise is applied. Experimental (squares), electric neuron simulation (diamonds) and the theory [29] (solid line). The horizontal axis represents noise: hydrodynamic in the case of the mechanoreceptors and electric in the case of the neuron model. Reproduced from [28] and [29].

stochastic time course [35]. The bistable perceptual process involved in the interpretation of ambiguous figures can be modeled by a noisy auto-associative neural network [33]. Using this system, the results indicate that SR can be demonstrated.

Another aspect is the human visual system that derives from threshold theory [31] and is closely related to the dithering effect [32]. Consider a system that is capable of transmitting single bits of information, each of which marks a threshold crossing. A visual realization of this is shown in Fig. 2, which was generated following the procedure in [31]. The original gray scale image of the popular 'Lena' used in signal processing is depressed beneath a threshold. Noise is added to the gray value in each pixel and the result compared to the threshold value. Pixels valued above the threshold are made white, the others black. Every pixel contains one bit of information, whether or not the threshold has been crossed. Fig. 2 shows the result of adding noise of three intensities, increasing from left to right. The optimal noise intensity in the center pane maximizes the information content. Note that the pictures shown in Fig. 2 only have the noise varying spatially; additional improvement in perceived picture quality can be gained by varying the noise temporally [31].

\section{Opinion Formation}

In the third example, SR is applied to the formation of public opinion between two parties. The formation of an individual's opinion is influenced by the presence of groups of people with the same or opposite opinion. The formation of the opinion changes over time. Thus, there is a small periodic force that changes the opinion from one side to another, which can be considered as a bistable system. If the influences from the groups of people with or without the same opinion are weak, then the change of opinion must be influenced by external information. The addition of collective noise is sufficient to induce a stochastic change of opinion. 

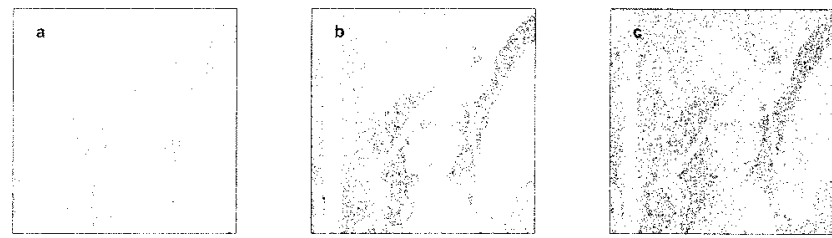

Fig. 2. Images are composed from a single 256 grayscale image. A random number from a Gaussian distribution with zero mean is added to the original gray value in every pixel. Thus, the noise is incoherent with that in all other pixels. The noise intensity is increased from left to right.

By performing calculations using the nonlinear sociological model of public opinion formation introduced by Weidlich, the signal-to-noise ratio can be determined. Determining the SNR as the collective noise is varied reveals that the formation of public opinion exhibits stochastic resonance [34].

\section{BISTABLE SYSTEMS}

Examples of bistable systems occur in areas such as physics [bistable super-conducting quantum interface device (SQUID) loops [29], bidirectional ring lasers [17]], biology (sensory systems [28], [30]), Earth sciences [climate [14], [29], [36]] and electronics (Schmitt trigger [16], tunnel diode [37], [38]). Here, a simple double well potential in a nonlinear system is described [16], [18], [23], [39].

A model of a one-dimensional nonlinear system that exhibits stochastic resonance is the damped harmonic oscillator with the Langevin equation of motion

$$
m \ddot{x}(t)+\gamma \dot{x}(t)=-\frac{d U(x)}{d x}+\sqrt{D} \xi(t)
$$

where the dot represents the time derivative [37]. This equation describes the motion of a particle of mass $m$ moving in the presence of friction $\gamma$. The restoring force is expressed as the gradient of some bistable or multi-stable potential function $U(x)$. In addition, there is an additive stochastic force $\xi(t)$ with intensity $D$ that has the statistical properties given in (7).

When $U(x)$ is a bistable potential, (1) may model several physical processes, ranging from the dynamics of a nonlinear elastic mechanical oscillator to the transient dynamics of a laser. A simple symmetric bistable potential has the form of a standard quartic

$$
U(x)=-a \frac{x^{2}}{2}+b \frac{x^{4}}{4} .
$$

If the system is heavily damped, the inertial $m \ddot{x}$ term can be neglected. Rescaling the system in (1) with the damping term $\gamma$ gives the stochastic Ginzburg-Landau equation

$$
\dot{x}(t)=-\frac{d U(x)}{d x}+\sqrt{D} \xi(t)
$$

which is frequently used to model nonequilibrium critical phenomena. For $a>0$, the potential is bistable, as shown in Fig. 3 . From simple algebra, there is an unstable state at $x=0$ and two stable states at $x_{s}= \pm \sqrt{a / b}$, separated by a barrier of height of $\Delta U=a^{2} / 4 b$ when the noise $\xi(t)$ is zero. The position of the particle $x(t)$ is considered to be the output of the system and has a power spectrum $S(\omega)$.

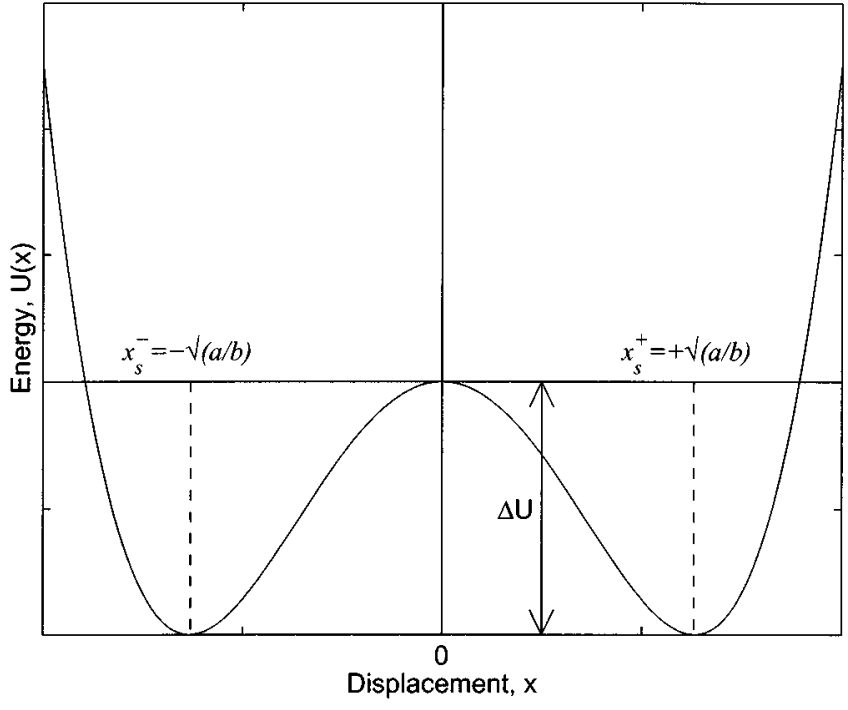

Fig. 3. Quartic bistable potential (2) without any noise with minima at $x_{s}$ and barrier height $\Delta U$.

Two examples [40] of nonlinear dynamic systems are the analog Hopfield neuron

$$
U(x)=\alpha x^{2}-\beta \ln (\cosh x)
$$

where the state point $x(t)$ denotes a cell membrane voltage and the SQUID loop

$$
U(x)=\alpha x^{2}-\beta \cos (2 \pi x)
$$

where $x(t)$ denotes the magnetic field flux in the loop.

By itself, the bistable system is stationary as described by the motion of the particle. That is, if the particle is in one of the two wells, it will stay there indefinitely. By adding a periodic signal, $A \sin \left(\omega_{s} t\right)$ to the bistable system, the dynamics are governed by the following equation

$$
\frac{d x}{d t}=\left(-\frac{d U(x)}{d x}+A \sin \left(\omega_{s} t\right)\right)+\sqrt{D} \xi(t) .
$$

The bistable potential, which is now time dependent, becomes

$$
\begin{aligned}
U(x, t) & =U(x)-A x \sin \left(\omega_{s} t\right) \\
& =-a \frac{x^{2}}{2}+b \frac{x^{4}}{4}-A x \sin \left(\omega_{s} t\right)
\end{aligned}
$$

where $A$ and $\omega_{s}$ are the amplitude and the frequency of the periodic signal, respectively.

Throughout this analysis, it is assumed that the signal amplitude is small enough that, in the absence of any noise, it is insufficient to force a particle to move from one well to another. It is also assumed that the signal period is longer than some characteristic intrawell relaxation time for the system.

Due to the presence of the modulating signal, the double well potential $U(x, t)$ is periodically tilted back and forth with the same frequency, $\omega_{s}=2 \pi / T_{s}$. Relating this to the potentials in Fig. 4, the effect is to weakly tilt the potential barriers to the right and left sides, respectively. In one period of the signal, the potential is cycled through Fig 4 (a)-(d). The maxima and minima of the signal correspond to when the potential barrier is at its lowest, which is in Fig. 4 (b) and (d). 
(a)

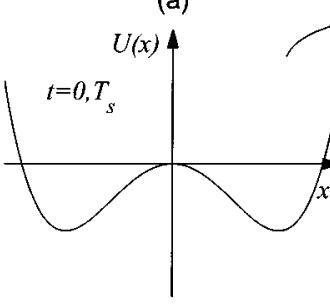

(c)

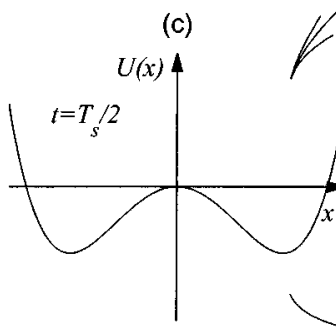

(b)

(d)
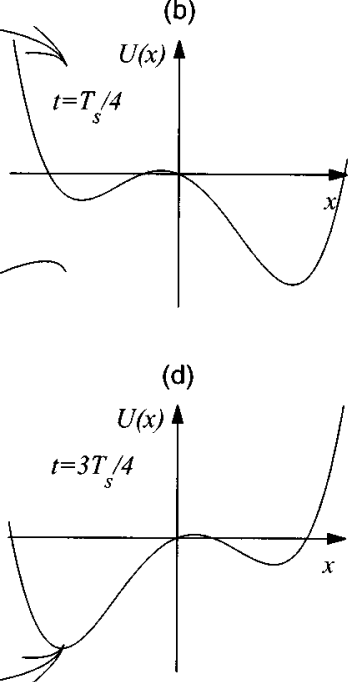

Fig. 4. Periodic driving signal causes the DWP to be tilted back and forth, antisymmetrically raising and lowering the left and right potential wells.

The quartic potential system shown in Fig. 4 is of particular interest because it represents the simplest bistable system in a continuous variable. It is seen that there are three fixed points, two stable and one unstable. All that is needed is a system with a cubic characteristic that has three operating points for some parameter. A tunnel diode with a load line defining the operating points would be an example of an electrical system that fits this requirement [37].

When considering the use of an electrical device to produce a bistable system, the stability of the operating points needs to be considered. By considering small perturbations, usually in the input current or voltage, the stationary state can be determined to be stable or unstable. It is concluded from [41] that there must be at least three stationary states if two stable states are to exist and for bistability to occur.

A natural simplification of the double-well problem is the discrete two-state system, in which the dynamical variable can take on only two discrete values. The Schmitt trigger is an example of such a discrete system. This is a useful system since its dynamics are well modeled as a two-state system, except for the switching between the two voltages which takes a finite amount of time. Since this switching occurs at rates far faster than any signal frequency of interest, it can, for all practical purposes, be considered as an ideal two-state system. An important feature of the Schmitt trigger is that it has hysteresis-that is there is a range of the input for which the circuit is bistable.

Another two-state system in common use is the threshold system. This is a simple system that produces an output voltage whenever the input exceeds the threshold value. In biological systems, it is referred to as a fire-and-reset model and works by sending a pulse of fixed width whenever the signal exceeds a certain threshold. Although there are two types of bistable systems, continuous and discrete, it is not obvious how to compare the results between them [24].

\section{QUANTIFYING SR}

The most common way to quantify SR is through the signal-to-noise ratio [29], although other methods can be used
[18], [32], [42]. The SNR is readily obtained from the output by forming the power spectrum, which measures the frequency content of a time series. For a sinusoidal signal, the power spectral density (PSD) shows a sharp peak located at the signal frequency on a broadband noise background; the SNR is the ratio of the strength of this peak to the noise level. The SNR in decibels is

$$
S N R \equiv 10 \log _{10}\left(\frac{S\left(\omega_{s}\right)}{B\left(\omega_{s}\right)}\right)
$$

where $S$ and $B$ represent the values of the output PSD of the peak and the background level, respectively. Note that the SNR must be taken at the fundamental frequency, that is, the frequency of the input sinewave.

The signature of stochastic resonance is that the output SNR rises sharply to a maximum at an optimal noise intensity and decreases gradually for larger noise intensities as randomization overrides the cooperative effect. The detailed shape of this curve depends on the signal frequency and other system parameters. As the noise intensity approaches zero, there is no output as there are no switching or threshold crossings.

\section{NOISE}

We used white Gaussian noise with mean and autocorrelation given respectively by

$$
\langle\xi(t)\rangle=0 \quad \text { and } \quad\left\langle\xi(t) \xi\left(t^{\prime}\right)\right\rangle=\delta\left(t-t^{\prime}\right) .
$$

This implies that $\xi(t)$ and $\xi\left(t^{\prime}\right)$ are statistically independent for $t \neq t^{\prime}$ and the angled brackets $\langle\cdot\rangle$ denote an ensemble average.

For our SR measurements noise was realized using a generator that simulates nearly white noise directly (WAVETEK model 132 VCG/Noise Generator). ${ }^{1}$ In the absence of such a generator it is also possible to generate white noise by band limiting the output of maximal length shift register with a low-pass filter that has a cut-off frequency well below the clock frequency of the register [43].

To measure the intensity of the noise, a simple averaging type ac voltmeter was used with the scale adjusted accordingly. This gave the noise in units of root mean squared volts $\left(V_{\text {rms }}\right)$.

Different types of noise, or the colored Ornstein-Uhlenbeck process, for example, affect the stochastic resonance and are examined in [44]-[46].

\section{CIRCUITS}

In this section, we consider four circuits that can be constructed using op amps.

A simple analysis of the Schmitt trigger reveals that the thresholds are symmetrically placed about the reference level, $V_{\text {ref }}$ as

$$
V_{\mathrm{TL}}=V_{\text {ref }}-L_{+}\left(\frac{R_{1}}{R_{2}}\right) \text { and } V_{\mathrm{TH}}=V_{\text {ref }}-L_{-}\left(\frac{R_{1}}{R_{2}}\right)
$$

where $L_{+}$are the supply voltages for the op amp. It is seen that the thresholds are controlled by positive feedback loop, formed

${ }^{1}$ As the cutoff frequency of the white noise from the WAVETEK generator, is a lot higher than the signal frequency, then for all intents and purposes we can consider this "ideal" white noise. 


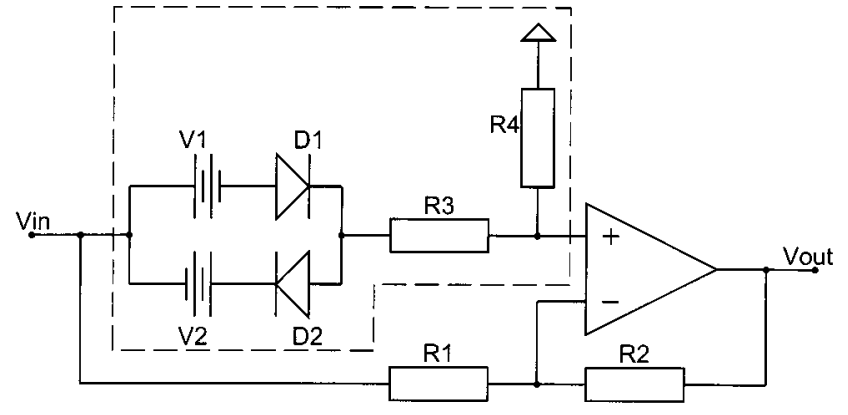

Fig. 5. OP amp circuit used to form an $\mathrm{N}$ shaped transfer characteristic. The portion enclosed in the dashed box was constructed with diodes and resistors only, powered from the same source as used for the op amp. This eliminated the need for extra voltage sources. This technique was also employed for the circuit shown in Fig. 6.

by $R_{1}$ and $R_{2}$. The values chosen for $R_{1}$ and $R_{2}$ can be arbitrary, chosen to give a hysteresis of desired width and can easily be changed to suit the output levels of the noise source.

A threshold device can be constructed from a single op amp, referred to as a level crossing circuit (LCC). The reference voltage (i.e., threshold) is supplied to the inverting input and an output is produced whenever the input is greater than this threshold. This functionality is similar to the biological neuron system [28], [47].

Real neurons exhibit a refractory period, or "dead time," after each firing event. Thus, at high noise levels, the firings become more frequent, but so do the refractory periods. This blanks out some of the subsequent noisy firings which keeps the SNR higher than it would otherwise be. In all biological systems there is unavoidable internal noise which is significant at low external noise but becomes masked at high external noise levels.

In our electrical LCC, the width of the pulse is determined by how long the input is above the threshold, but is quantized in the digital simulations. A large proportion of papers reporting level crossing circuits differ in that they output a pulse of fixed width whenever the threshold is crossed in the increasing direction [48], [49]. The theory for our nonfixed pulse width LCC can be found in [50], [51].

Adding some nonlinear elements in the form of diodes to an op amp circuit, characteristics can be produced that have potentially bistable nature. Two instances of these circuits we developed are discussed. The circuit that produces an $\mathrm{N}$ shaped transfer characteristic curve is shown in Fig. 5 and its characteristic in Fig. 7. Intuitively, it can be seen that the $\mathrm{N}$ shape transfer characteristic only modulates the input signal as it is piecewise linear. The circuit is not bistable, which is one of the requirements for SR to exist.

To produce an $\mathrm{S}$ shaped transfer characteristic, nonlinear feedback is required. The resulting circuit is shown in Fig. 6 where it is noted that the nonlinear elements are placed in the feedback loop. The characteristics of the circuit are shown in Fig. 7.

\section{RESULTS}

The S curve op amp, LCC, and Schmitt trigger circuits were digitally simulated, built, and experimentally tested. The SNR was plotted at the fundemental frequency to show evidence of SR.

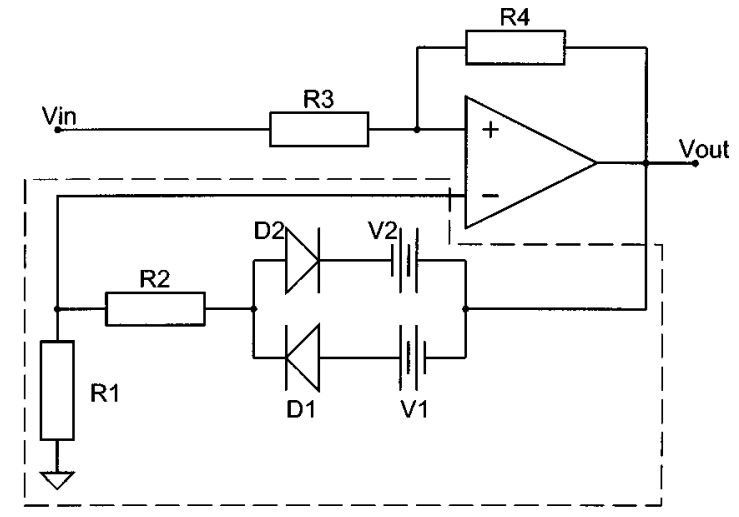

Fig. 6. OP amp circuit used to form an $\mathrm{S}$ shaped transfer characteristic. This has the nonlinear components in the feedback path.

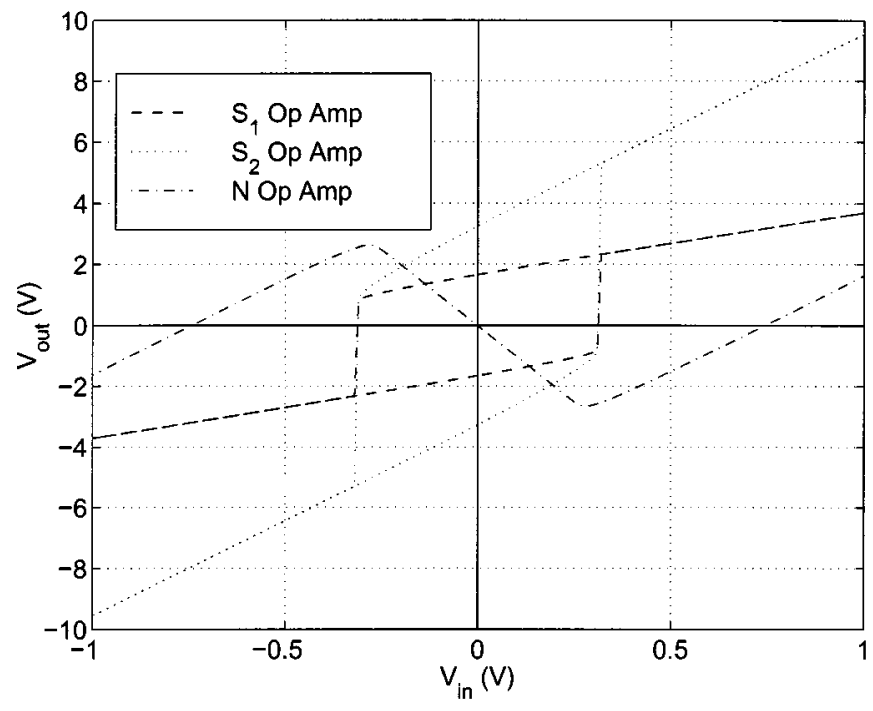

Fig. 7. Transfer characteristic of the $\mathrm{N}$ and two $\mathrm{S}$ shaped op amp circuits. (The horizontal scale needs to be multiplied by 10 for the $\mathrm{N}$ op amp).

The signal-to-noise ratio has been defined as the relationship between the output signal and the input noise at the signal frequency as described by (6). The spectrum analyzer (HEWLETT PACKARD 35665A Dynamic Signal Analyzer) was set to give the PSD in units of $V_{\mathrm{rms}}^{2} / \mathrm{Hz}$. The noise, which is measured by the multimeter in rms, must have the same dimensions. It was converted to the same units as the signal output PSD by use of (9), where BW is the noise bandwidth of the noise generator

$$
\mathrm{PSD}_{\text {noise }}=\frac{V_{\mathrm{rms}}^{2}}{\mathrm{BW}}
$$

Fig. 8 shows the results of three subthreshold signals with varying amplitudes fed into the Schmitt trigger circuit. The first signal $\left(1.8 V_{\mathrm{pp}}\right)$ was set with an amplitude just below the threshold, the second $\left(1.45 V_{\mathrm{pp}}\right)$ at about three quarters of the threshold, and the third $\left(1.0 V_{\mathrm{pp}}\right)$ at about half the threshold. This figure shows the SR behavior for the three signal amplitudes. The further the signal from the threshold, the more noise that is required to cause transitions; hence, the amount of improvement gained by SR decreases as the signal to threshold distance increases. 


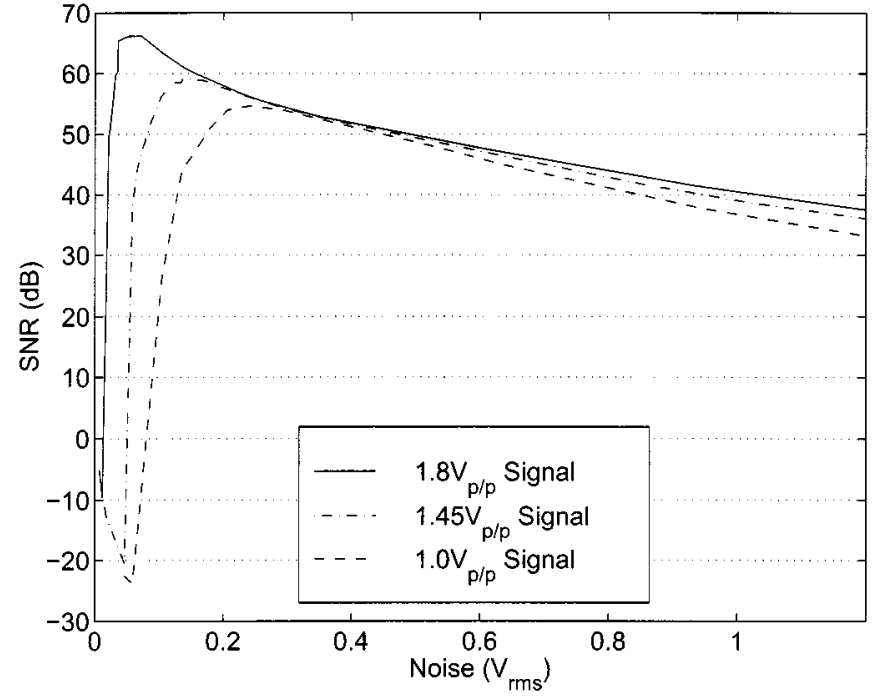

Fig. 8. SNR for different signal amplitudes in the Schmitt trigger circuit. The thresholds were placed at $\pm 1.0 \mathrm{~V}$ giving a hysteresis of $2.0 \mathrm{~V}$. The highest signal $\left(1.8 V_{\mathrm{pp}}\right)$ was chosen so that transitions would occur with only the slightest noise, whereas the lowest signal $\left(1.0 V_{\mathrm{pp}}\right)$ was about half of the hysteresis.

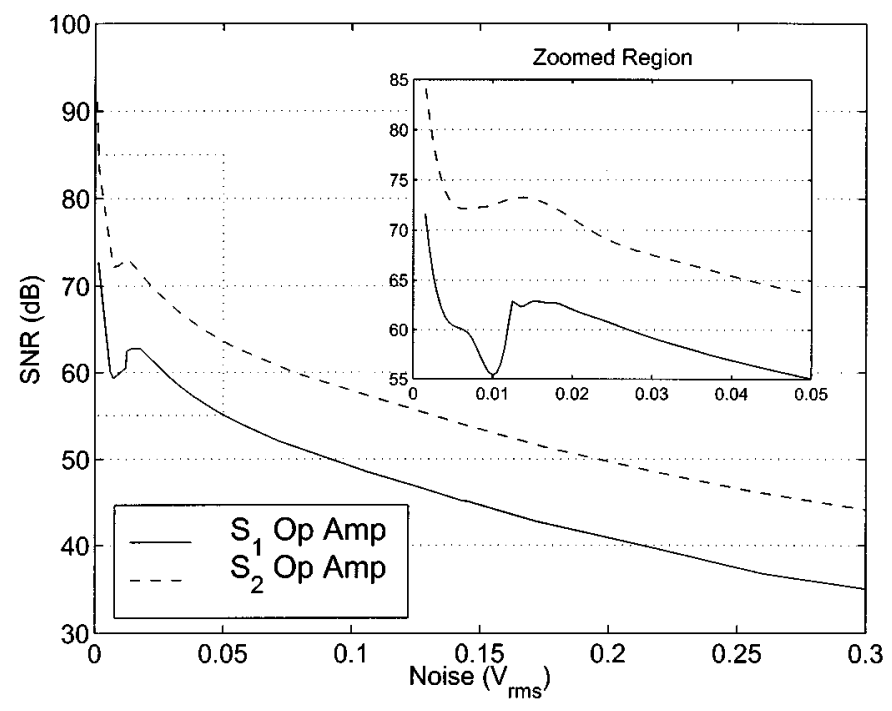

Fig. 9. SNR of the two $\mathrm{S}$ shape op amp circuits. The characteristics of the circuits are shown in Fig. 7. The input signal was $0.6 V_{\mathrm{pp}}$. To highlight the SR peaks, a zoomed in region is shown in the inset.

It is also noted from Fig. 8 that when the noise is initially increased from zero, there are no transitions. This is due to the input signal and the added noise never crossing the threshold. The effect is that there is an increased noise intensity for no increase in output signal, thus the SNR decreases. Once the noise increases enough to encourage transitions to occur, the SNR starts to increase sharply to the characteristic SR peak.

Results for two $\mathrm{S}$ shape transfer characteristic circuits that have the same threshold values, but varying slopes are shown in Fig. 9. The second circuit $\left(S_{2}\right)$ has twice the slope of the first circuit $\left(S_{1}\right)$. There was the initial decrease in SNR for low noise intensity, followed by a rather small SR peak. The reduced SR peak is due to the fact that when the slope of the $S$ curve is increased, the nonlinearity of the circuit is reduced. Hence, the circuit starts to behave according to linear response theory

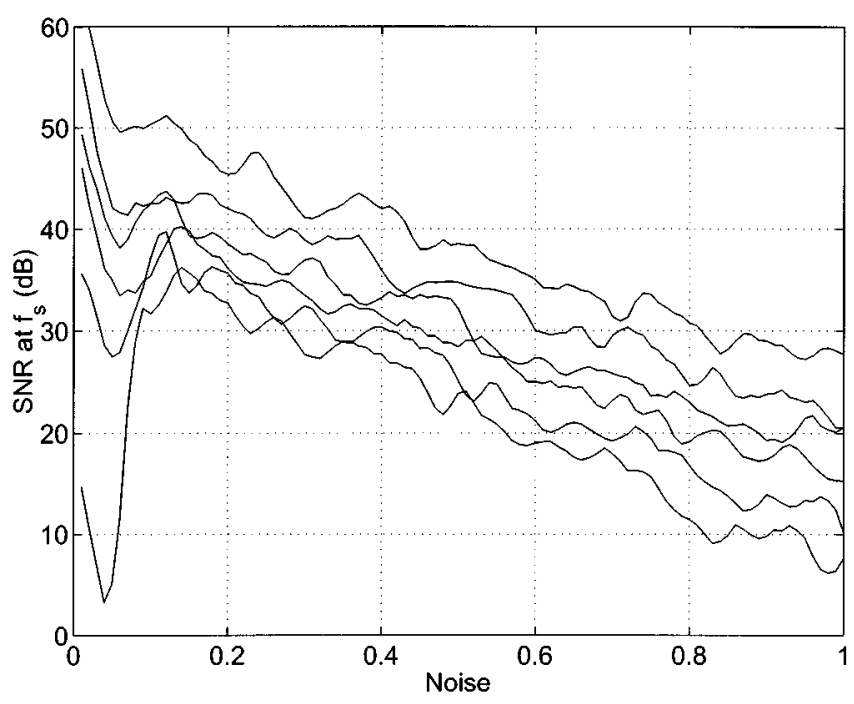

Fig. 10. Results as the noise is increased for varying the slopes of $\mathrm{S}$ shaped curves. The bottom to top lines are respectively ST, $S_{a}$ to $S_{e}$. The curves have been smoothed so the different lines can be easily distinguished.

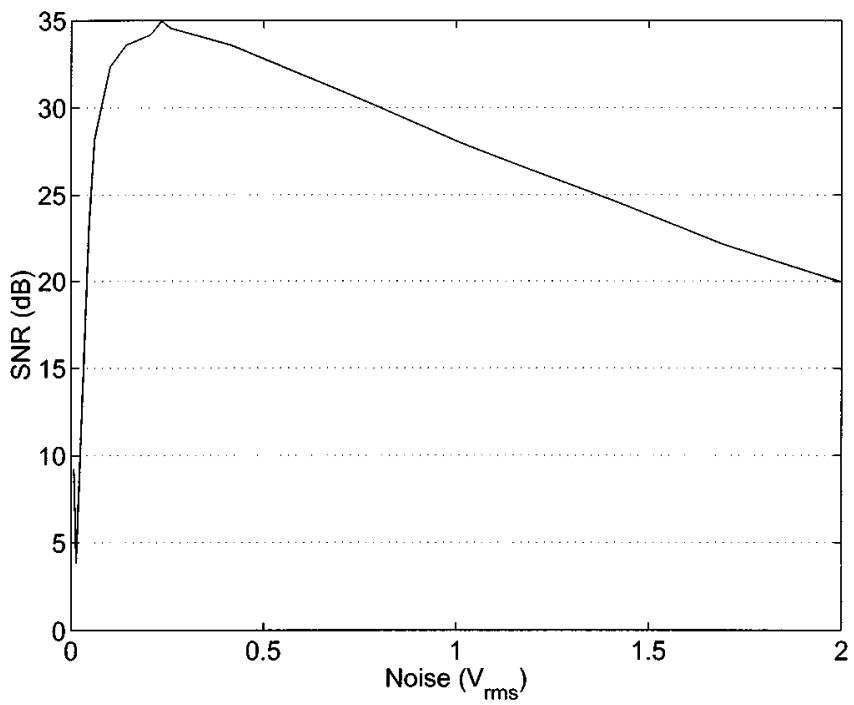

Fig. 11. SNR of a level crossing system that is commonly used to model sensory systems. The signal amplitude is only a small amount below the threshold and hence there is only a small amount of decrease in the SNR for low noise values.

where the output SNR is proportional to the input SNR and thus any improvement gained by SR is lost.

To explore this further, more $\mathrm{S}$ shape curves were generated and simulated. This shows more clearly the effects on the SNR caused by varying the slopes of S shaped curves. Starting from the Schmitt trigger (ST) curve, which has zero slope, the slopes were progressively increased from $S_{a}$ to $S_{e}$. Fig. 10 shows the SNR as the noise is increased. The bottom to top lines are respectively ST, $S_{a}$ to $S_{e}$. We can see for the ST curve, the SR peak is the largest, but once the slope of the characteristic increases, even only by a little, the SR peak is vastly reduced. Once the slope of the characteristic exceeds a certain level, the SR peak disappears and the SNR monotonically decreases as the noise is increased. There is a tradeoff between the magnitude of the SR peak and the overall SNR and thus it would be expected that a characteristic with zero slope would have the best SR peak for 
TABLE I

GAIN IN SNR THAT IS OFFERED BY EACH CIRCUIT

\begin{tabular}{lcc}
\hline \hline Circuit Type & Name & Improvement (dB) \\
\hline Schmitt Trigger & $\mathrm{ST}$ & 32.9 \\
\hline & $\mathrm{S}_{\mathrm{a}}$ & 12.3 \\
& $\mathrm{~S}_{\mathrm{b}}$ & 6.8 \\
& $\mathrm{~S}_{\mathrm{c}}$ & 5.6 \\
S Op Amp & $\mathrm{S}_{\mathrm{d}}$ & 2.1 \\
& $\mathrm{~S}_{\mathrm{e}}$ & 1.6 \\
\hline Level Crossing Circuit & & \\
(Variable width pulse) & $\mathrm{LCC}$ & 19.8 \\
\hline & $\mathrm{LCC}_{3 \%}$ & 20.2 \\
& $\mathrm{LCC}_{15 \%}$ & 26.5 \\
& $\mathrm{LCC}_{30 \%}$ & 31.1 \\
Level Crossing Circuit & 33.5 \\
(Fixed width pulse) & $\mathrm{LCC}_{45 \%}$ & 32.9 \\
& $\mathrm{LCC}_{60 \%}$ & 29.7 \\
\hline \hline & $\mathrm{LCC}_{75 \%}$ & \\
\hline
\end{tabular}

the SNR curve. The Schmitt trigger can be considered to have zero slope and its SNR shows the largest SR peak, as seen in Fig. 10.

The level crossing circuit is different from the other circuits investigated as it is a threshold system. The signal-to-noise ratio of the LCC is shown in Fig. 11. The peak of the LCC is not as sharp as the previous two SNR curves (Figs. 8 and 9), but it is higher for large noise intensities. This implies that this circuit is more immune to noise, possibly why nature has adapted this technology for use in biological systems. The SNR of the level crossing circuit and other threshold systems [28], [29] is quite comparable, seen by the similar shaped curves in Figs. 1 and 11.

The amount of gain achievable in SNR is inherently dependent on the circuit characteristics. From simulations, the gain for each of the circuits is tabulated in Table I. The gain $(\mathrm{dB})$ has been defined as the improvement on SNR due to SR, that is the difference between the valley at low noise intensities and the SR peak. The percentage value shown in the subscript of LCC in the Name column of Table I indicates the pulse width to that of the signal period, $T_{s}$. It is clear from Table I that as the slope of the $\mathrm{S}$ shaped characteristics increases, the amount of gain in the SNR steadily decreases. Considering the LCCs, the gain in SNR of the variable width pulse is comparable to that of the LCC with a width of approximately two to three percent of $T_{s}$. The gain in SNR offered by SR increases with pulse width until the width reaches about fifty percent of $T_{s}$ where the SNR then starts to decrease.

As stochastic resonance has been demonstrated in different circuits, it is interesting to compare the time and the frequency domain responses during the process of increasing the noise. Using the Schmitt trigger circuit with a signal of $1.45 V_{\mathrm{pp}}$,

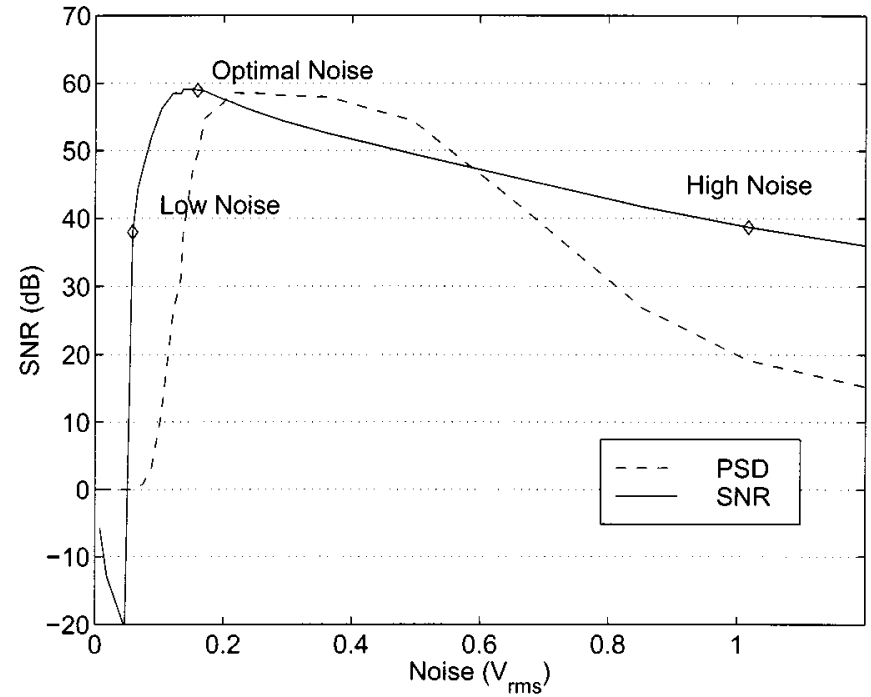

Fig. 12. SNR and the PSD of the $1.45 V_{\mathrm{p} / \mathrm{p}}$ signal for the Schmitt trigger indicating the low, optimal and high noise positions. This was extracted from Fig. 8.

snap shots were taken for the three positions shown on the SNR curves in Fig. 12. Fig. 13 shows the low, optimal, and high noise positions in parts (a), (b), and (c), respectively. While observing stochastic resonance in the frequency domain, it is noted that there is no actual increase in signal amplitude in the time domain. The amplitude of the signal is constant although the SNR is changing. In traditional resonating circuits it was the amplitude of the output signal that peaked; here it is the coherency of the signal. This increases the SNR as required, but does not actually increase the output signal strength. The SR phenomenon could more appropriately be described as a noise induced signal-to-noise ratio enhancement, not such an elegant expression, but a more accurate one [39].

Comparing the PSD and the SNR in Fig. 12, it is interesting to note that the SNR does not reach a maximum at the same amplitude as the PSD does. The reason is that the rate of increase of the signal component is far greater than that of the noise. In other words, the SNR has its maximum where the PSD has its greatest rate of change.

\section{APERIODIC SR}

A large proportion of work in SR has been limited to systems with periodic stimulus. Although it has served useful in many areas, the applicability of SR to practical applications is limited. This is due to many real world stimuli being aperiodic.

This limitation leads to the concept of ASR, first coined by Collins et al. [52]. ASR introduces another hindrance, namely, how to measure it. The SNR measure essentually assesses the coherence of the system response with the input signal. This metric is inappropriate for systems with aperiodic inputs.

A cross correlation based measure was introduced [52] that considers the correlation between the stimulus signal and the system response. This is termed the power norm $C_{0}$ and is given by

$$
C_{0}=\langle[S(t)-\overline{S(t)}][R(t)-\overline{R(t)}]\rangle
$$



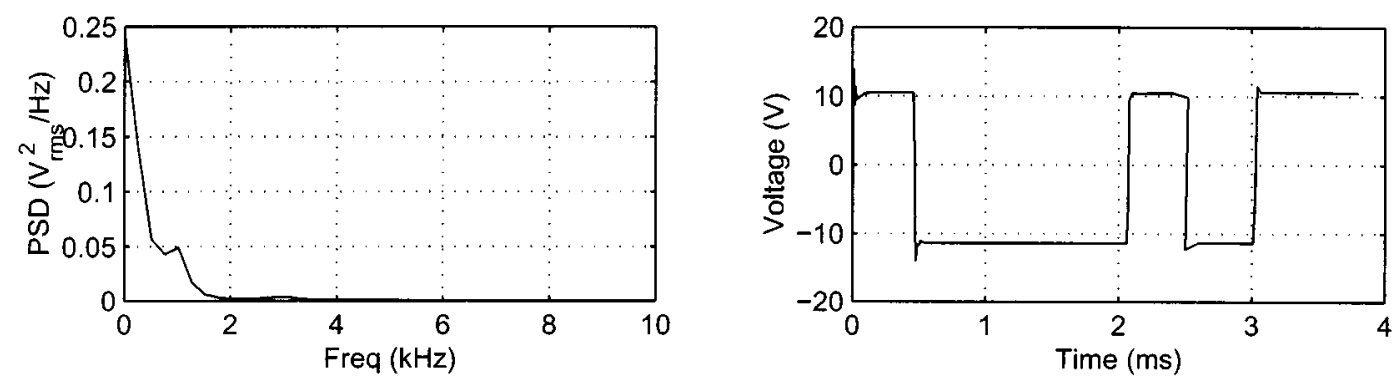

(a)
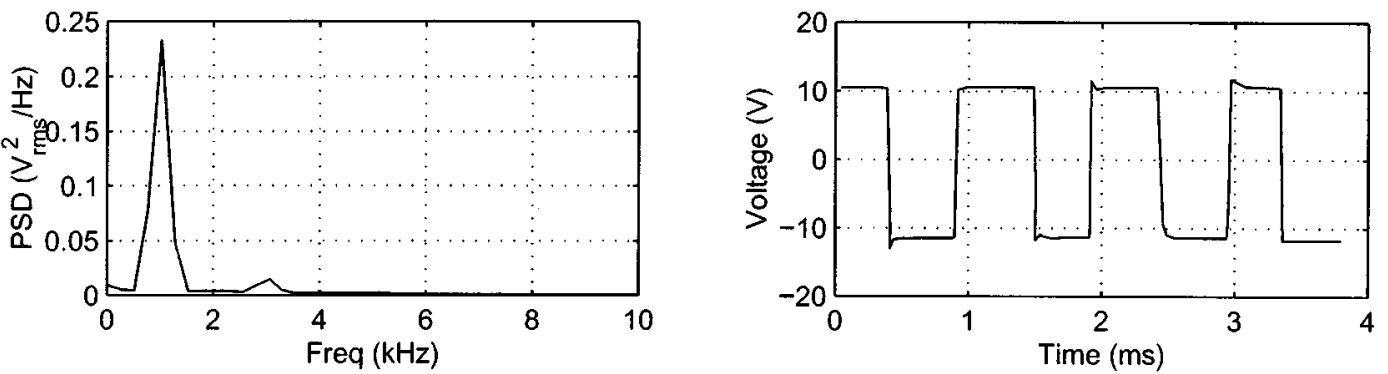

(b)
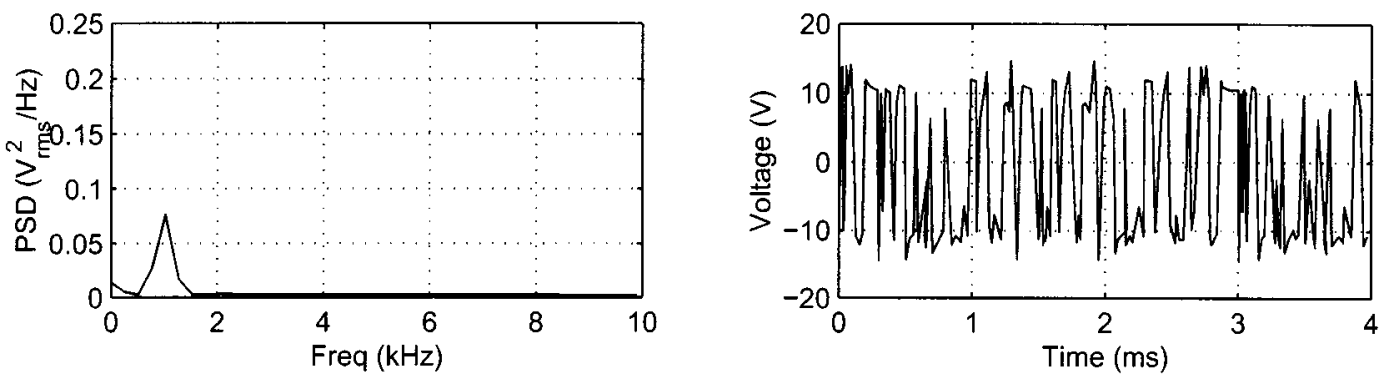

(c)

Fig. 13. Comparing the frequency and time domain as the noise is increased. In (a), only low noise has been added, causing intermittent transitions. Adding optimal amount of noise in (b) causes regular transitions, and adding high noise in (c) causes the transitions to be dominated by the noise.

where $R(t)$ is the mean firing rate constructed from the system output and the overbar denotes a temporal average. The normalized power norm $C_{1}$ is given by

$$
C_{1}=\frac{C_{0}}{\left\langle[S(t)-\overline{S(t)}]^{2}\right\rangle^{1 / 2}\left\langle[R(t)-\overline{R(t)}]^{2}\right\rangle^{1 / 2}} .
$$

These measures assume the peak in the input-output cross-correlation occurs at a time lag of zero. However, in certain systems a lag may exist between the stimulus and response. In this case, one should use the peak in the input-output cross-correlation function.

An aperiodic signal was formed by convoluting a 10-s Hanning window filter was convolved with colored noise having correlation time of $20 \mathrm{~s}$. This has the effect of smoothing the signal like a low-pass filter, ensuring the time scale of the signal is much greater then that of the noise [52], [53]. This signal is amplified and shifted to give zero mean and variance $1.5 \times 10^{-5}$. The threshold voltage for the LCC was set to 0.1 V. The results shown in Fig. 14 clearly show ASR is exhibited. Thus, using the simplest nonlinear system the input-output correlation can be improved for any signal with addition of noise.
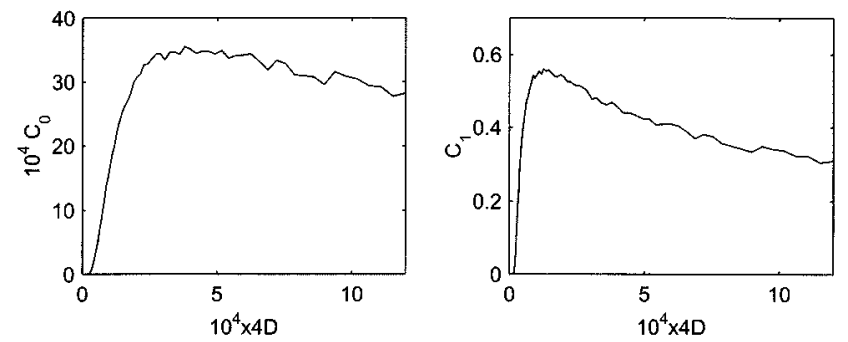

Fig. 14. Numerical simulations of the LCC system with an aperiodic signal showing the correlations $C_{0}$ and $C_{1}$.

A criticism with using $C_{1}$ from (11) is that it is a measure for systems where the dynamics are linear [54]. Although it is shown that (noise induced) linearization occurs at large noise amplitudes [55], it is probably not satisfactory when the systems used are designed to have nonlinear dynamics. An information theoretical measure initiated by Stocks et al. [56], [57] would be much more appropriate than the linear signal processing technique such as the cross-correlation measure $C_{1}$.

By utilizing a summing network of $N$ threshold devices, Stocks defines the average mutual information (AMI) that is transmitted through the network as

$$
A M I=H(y)-H(y \mid x)
$$


where $H(y)$ is the information content of the output $y(t)$ and $H(y \mid x)$ is the conditional entropy of the output given the input. Using the system and $A M I$ measure, Stocks describes a suprathreshold stochastic resonance (SSR), where the signal is greater that the threshold. Providing there is more that one element in the network, the maximum transmitted information is shown to occur at some nonzero noise intensity. Improvements to the AMI can be made by adjusting the distribution of thresholds in the $N$ devices [58]. This shows an important result, when the thresholds are set optimally for a certain signal and noise distribution, the addition of noise only reduced the $A M I$. This is similar to the well known suprathreshold systems where noise only degrades the response [59]-[61].

There are also other methods and systems besides the one just investigated that have been analyzed in terms of information theory. However, they are generally defined similarly to (12) [40], [53], [59], [61]-[64].

In some cases, the entropy based measure does not fully characterize the bandwidth the system. It assumes that the bandwidth is independent of noise, which is not always true. The appropriate measure should be based on Shannon's channel capacity. This has recently been shown for a threshold system [65], where the channel capacity also shows a noise induced improvement in performance.

\section{APPLiCATIONS}

In most physical systems, the signal and noise are fixed with a threshold at the receiver being variable. Even though noise could be added at the receiver in order to get an increase in SNR, it is not going to be as good as using the minimal amount of noise possible and changing the threshold. Rather than thinking in terms of finding the optimal noise value for a given threshold, it may be more useful to think of SR in terms of finding the optimal threshold for given noise. Thus, in order for SR to be useful for a physical application, we ideally need a system with a threshold, but where the value of the threshold is not critical to the output.

This naturally lends itself to a binary threshold system with the output being in either one of two states. It has been demonstrated that the information capacity in an asymmetric binary channel reaches at a maximum with the addition of noise [61] and the effects of varying the thresholds are also shown.

Another area of interest of SR is in signal processing where weak signals are embedded in noise. SR can be used to enhance the signal-to-noise ratio of the output making it useful in a receiver. These signals need not be periodic as SR has been displayed to be present in nonperiodic signals [18].

Since 1992, our group has worked on a model of the insect visual system to detect motion [66]-[68]. Motion is determined by detecting edges in the image plane and comparing them over time. In this case, the signals from the insect vision sensor can be noisy. Edges that are determined by comparing different pixels to a threshold could use SR to enhance their detection. Since the noise varies spatially as well as temporally, a large improvement in the image quality is expected [31]. Such schemes may prove critical in the initiative to perform insect vision processing in the millimeter-wave region [69].
It is seen that SR requires the threshold to be carefully set, thus not making it very robust for engineering applications. A variation of SR is "suprathreshold stochastic resonance (SSR)" [56], where the threshold is set below the peak of the stimulus. It is shown that a single suprathreshold element can perform as well as a network of suprathreshold elements [54]. This leads us to believe that SR may be applicable where a poor choice of fixed threshold has been made; otherwise, the threshold can simply be varied for optimum performance in the suprathreshold regime. Hence, to create a more robust motion detection scheme, SSR may be the answer.

\section{Concluding Remarks}

We have reviewed a number of circuits that demonstrate stochastic resonance. SR is more effective when the nonlinear system does not modulate any of the input noise on to the output signal. In other words, the more nonlinear the system the larger the gain in SNR, as seen in Table I. A system which has discrete set states, like the Schmitt trigger or a level crossing circuit, has better SR peak than a system with gain, like the S shaped transfer circuit. This is due to the increased slope of the $\mathrm{S}$ shaped transfer function curves reducing the nonlinearity.

The proximity of the signal to the threshold is also an important factor. As the signal amplitude becomes smaller (i.e., the signal to threshold distance becomes larger), the size of the SR peak in the SNR is reduced as more noise is required to cause transitions. We have highlighted and clarified that the SR peak in the SNR curve does not occur at the same noise value as the maximum for the PSD curve. Finally, we have presented simple nonlinear op-amp circuits suitable for demonstrating and measuring SR in the laboratory.

Future work should concentrate on utilizing SSR to create a more robust system. In particular, for the motion detection scheme and a system of parallel thresholding elements could utilize SSR in detecting edges in noisy images. As a quantitative measure, the information theoretical metric is a natural choice.

\section{ACKNOWLEDGMENT}

The authors would like to thank A. J. Parfitt for helpful comments and manuscript corrections.

\section{REFERENCES}

[1] A. N. Netravali and B. G. Haskell, Digital Pictures: Representation and Compression. New York: Plenum, 1988.

[2] C. J. Kikkert and A. Biddeli, "Hardware additive dither for analogue to digital converters," in Proc. IREE 14th Australian Microelectronics Conf., Melbourne, Vic., Australia, Sept 29.-Oct 1. 1997, pp. 156-161.

[3] M. Bier, "Brownian ratchets in physics and biology," Contemp. Phys., vol. 38, no. 6, pp. 371-379, 1997.

[4] A. Rosato, K. J. Strandburg, F. Prinz, and R. H. Swendsen, "Why the Brazil nuts are on top: Size segregation of particulate matter by shaking," Phys. Rev. Lett., vol. 58, no. 10, pp. 1038-1040, 1987.

[5] H. M. Jaeger, S. R. Nagel, and R. P. Behringer, "Granular solids, liquids and gases," Rev. Modern Phys., vol. 68, no. 4, pp. 1259-1273, Oct. 1996.

[6] H. A. Makse, S. Havlin, P. R. King, and H. E. Stanley, "Spontaneous stratification in granular mixtures," Nature, vol. 386, pp. 379-382, 1997.

[7] C. M. Bishop, Neural Networks for Pattern Recognition. London, U.K.: Oxford, 1996, ch. 9, pp. 346-349.

[8] G. P. Harmer, D. Abbott, and P. G. Taylor, "The paradox of Parrondo's games," Proc. R. Soc. A, vol. 456, no. 1994, pp. 247-260, 2000. 
[9] G. P. Harmer and D. Abbott, "Parrondo's paradox: Losing strategies cooperate to win," Nature, vol. 402, pp. 864-864, 1999.

[10] G. J. Chaitin, The Unknowable. New York: Springer-Verlag, 1999.

[11] P. C. W. Davies, "Vacuum viscosity and quantum noise: From atoms to galaxies," in Second Int. Conf. Unsolved Problems of Noise and Fluctuations, D. Abbott and L. B. Kish, Eds., Adelaide, Australia, 2000, pp. $16-31$.

[12] R. Benzi, "Unpublished Short Communication,” NATO Int. Sch. Climatology, Erice, Italy, 1980

[13] R. Benzi, G. Parisi, A. Sutera, and A. Vulpiani, "Stochastic resonance on climate change," Tellus, vol. 34, no. 1, pp. 10-16, 1982.

[14] C. Nicolis, "Stochastic aspects of climate transitions response to a periodic forcing," Tellus, vol. 1, no. 34, pp. 1-9, 1982.

[15] R. Benzi, A. Sutera, and A. Vulpiani, "The mechanism of stochastic resonance," J. Phys. A, vol. 14, pp. L453-457, 1981.

[16] S. Fauve and F. Heslot, "Stochastic resonance in a bistable system," Phys. Lett., vol. 97A, no. 1, pp. 5-7, 1983.

[17] B. McNamara, K. Wiesenfeld, and R. Roy, "Observation of stochastic resonance in a ring laser," Phys. Rev. Lett., vol. 60, no. 25, pp. 223-287, 1988 .

[18] L. Gammaitoni, P. Hänggi, P. Jung, and F. Marchesoni, "Stochastic resonance," Rev. Mod. Phys., vol. 70, no. 1, pp. 223-287, 1998.

[19] D. G. Luchinsky, R. Mannella, P. V. E. McClintock, and N. G. Stocks, "Stochastic resonance in electrical circuits-I: Conventional stochastic resonance," IEEE Trans. Circuits Syst. II, vol. 46, no. 9, pp. 1205-1214, 1999.

[20] _ - "Stochastic resonance in electrical circuits-II: Nonconventional stochastic resonance," IEEE Trans. Circuits Syst. II, vol. 46, pp. 1215-1224, Sept. 1999.

[21] L. Gammaitoni, F. Marchesoni, E. Menichella-Saetta, and S. Santucci, "Stochastic resonance in bistable systems," Phys. Rev. Lett., vol. 62, no. 4, pp. 349-351, 1989

[22] R. F. Fox and Y. Lu, "Analytical and numerical study of stochastic resonance," Phys. Rev. E, vol. 48, no. 5, pp. 3390-3398, 1993.

[23] P. Jung and P. Hänggi, "Amplification of small signals via stochastic resonance," Phys. Rev. A, vol. 44, no. 12, pp. 8032-8042, 1991.

[24] B. McNamara and K. Wiesenfeld, "Theory of stochastic resonance," Phys. Rev. A, vol. 39, no. 9, pp. 4854-4869, 1989.

[25] J. M. G. Vilar and J. M. Rubí, "Stochastic multiresonance," Phys. Rev. Lett., vol. 78, no. 15, pp. 2882-2885, Apr. 1997.

[26] F. Jaramillo and K. Wiesenfeld, "Mechanoelectrical transduction assisted by Brownian motion: A role for noise in the auditory system," Nature Neurosci., vol. 1, no. 5, pp. 384-388, 1998.

[27] I. C. Bruce, M. W. White, L. S. Irlicht, S. J. O'Leary, S. Dynes, E. Javel, and G. M. Clark, "A stochastic model of the electrically stimulated auditory nerve: Single-pulse response," IEEE Trans. Biomed. Eng., vol. 46, pp. 617-629, June, 1999.

[28] F. Moss and K. Wiesenfeld, "The benefits of background noise," Sci. Amer., pp. 50-53, 1995.

[29] K. Wiesenfeld and F. Moss, "Stochastic resonance and the benefits of noise: From ice ages to crayfish and SQUID's," Nature, vol. 373, pp. 33-36, 1995

[30] J. K. Douglas, L. Wilkens, E. Pantazelou, and F. Moss, "Noise enhancement of information transfer in crayfish mechanoreceptors by stochastic resonance," Nature, vol. 365, pp. 337-339, 1993.

[31] E. Simonotto, M. Riani, S. Charles, M. Roberts, J. Twitty, and F. Moss, "Visual perception of stochastic resonance," Phys. Rev. Lett., vol. 78, no. 6, pp. 1186-1189, 1997.

[32] L. Gammaitoni, "Stochastic resonance and the dithering effect in threshold physical systems," Phys. Rev. E, vol. 52, no. 5, pp. 4691-4698, Nov. 1995.

[33] M. Riani and E. Simonotto, "Stochastic resonance in the perceptual interpretation of ambiguous figures: A neural network model," Phys. Rev. Lett., vol. 72, no. 19, pp. 3120-3123, 1994.

[34] P. Babinec, "Stochastic resonance on the Weidlich model of public opinion formation," Phys. Lett. A, vol. 225, pp. 179-181, 1997.

[35] M. Riani and E. Simonotto, "Periodic perturbation of ambiguous figure: A neural-network model and a nonsimulated experiment," $\mathrm{Nuovo} \mathrm{Ci}$ mento, vol. 17D, no. 7-8, pp. 903-913, 1995.

[36] R. Monastersky, "Staggering through the ice ages: What made the planet careen between climate extremes?," Sci. News, vol. 146, no. 5, pp. 74-76, 1994.

[37] E. Lanzara, R. N. Mantegna, B. Spagnolo, and R. Zangara, "Experimental study of a nonlinear system in the present of noise: The stochastic resonance," Amer. J. Phys., vol. 65, no. 4, pp. 341-349, 1997.

[38] R. N. Mantegna and B. Spagnolo, "Stochastic resonance in a tunnel diode," Phys. Rev. E, vol. 49, no. 3, pp. R1792-1795, 1994.
[39] R. F. Fox, "Stochastic resonance in a double well," Phys. Rev. A, vol. 39, no. 8 , pp. $4148-4153,1989$.

[40] A. R. Bulsara and L. Gammaitoni, "Tuning into noise," Phys. Today, vol. 49, no. 3, pp. 39-45, 1996

[41] A. Wacker and E. Schöll, "Criteria for stability in bistable electrical devices with S- or Z-shaped current voltage characteristic," J. Appl. Phys., vol. 78, no. 12, pp. 7352-7357, 1995.

[42] K. Loerincz, Z. Gingl, and L. B. Kiss, "A stochastic resonator is able to greatly improve signal-to-noise ratio," Phys. Lett. A, vol. 224, no. 1-2, pp. 63-67, 1996.

[43] P. Horowitz and W. Hill, The Art of Electronics. New York: Cambridge Univ. Press, 1980.

[44] L. Gammaitoni, E. Menichella-Saetta, S. Santucci, F. Marchesoni, and C. Presilla, "Periodically time-modulated bistable systems: Stochastic resonance," Phys. Rev. A, vol. 40, no. 4, pp. 2114-2119, 1989.

[45] P. Hänggi, P. Jung, C. Zerbe, and F. Moss, "Can colored noise improve stochastic resonance?,” J. Stat. Phys., vol. 70, pp. 25-51, 1993.

[46] R. N. Mantegna and B. Spagnolo, "Stochastic resonance in a tunnel diode in the presence of white noise or colored noise," Nuovo Cimento, vol. 17D, no. 7-8, pp. 873-881, 1995 .

[47] Z. Gingl, L. B. Kiss, and F. Moss, "Non-dynamical stochastic resonance: Theory and experiments with white and arbitrarily colored noise," Europhys. Lett., vol. 29, no. 3, pp. 191-196, 1995.

[48] - "Non-dynamical stochastic resonance: Theory and experiments with white and various colored noises," Nuovo Cimento, vol. 17D, no. 7-8, pp. 795-802, 1995.

[49] K. Wiesenfeld, "Stochastic resonance on a circle," Phys. Rev. Lett., vol. 72 , no. 14 , pp. $2125-2129,1994$

[50] F. Chapeau-Blondeau and X. Godivier, "Theory of stochastic resonance in signal transmission by static nonlinear systems," Phys. Rev. E, vol. 55, no. 2, pp. 1478-1495, 1997.

[51] X. Godivier and F. Chapeau-Blondeau, "Noise-assisted signal transmission in a nonlinear electronic comparator: Experiment and theory," Signal Process., vol. 56, no. 3, pp. 293-303, 1997.

[52] J. J. Collins, C. C. Chow, and T. T. Imhoff, "Aperiodic stochastic resonance in excitable systems," Phys. Rev. E, vol. 52, no. 4, pp. 3321-3324, 1995.

[53] C. Heneghan, C. C. Chow, J. J. Collins, T. T. Imhoff, S. B. Lowen, and M. C. Teich, "Information measures quantifying aperiodic stochastic resonance," Phys. Rev. E, vol. 54, no. 3, pp. 2228-2231, 1996.

[54] N. G. Stocks and R. Mannella, "Suprathreshold stochastic resonance in a neuronal network model: A possible strategy for sensory coding," in Future Directions for Intelligent Systems and Information Sciences, N. Kosabov, Ed. New York: Physica-Verlag, 2000, pp. 236-247.

[55] D. R. Chialvo, A. Longtin, and J. Müller-Gerking, "Stochastic resonance in models of neuronal ensembles," Phys. Rev. E, vol. 55, no. 2, pp. 1798-1808, 1997.

[56] N. G. Stocks, "Suprathreshold stochastic resonance," in Stochastic and Chaotic Dynamics in the Lakes, D. S. Broomhead, E. A. Luchinskaya, P. V. E. McClintock, and T. Mullin, Eds. Ambleside, UK: AIP, 2000, vol. 502, pp. 415-421.

[57] _ _ "Suprathreshold stochastic resonance in multilevel threshold systems," Phys. Rev. Lett., vol. 84, no. 11, pp. 2310-2313, 2000.

[58] _ _ "Optimising information transmission in model neuronal ensembles," in Stochastic Processing in Physics, Chemistry and Biology, J. A. Freund and T. Poschel, Eds. Berlin, Germany: Springer-Verlag, 2000, vol. 557, Lecture Notes in Physics, pp. 150-159.

[59] M. DeWeese and W. Bialek, "Information flow in sensory neurons," $I l$ Nuovo Cimento D, vol. 17, no. 7-8, pp. 733-741, 1995.

[60] A. R. Bulsara and A. Zador, "Threshold detection of wideband signals: A noise-induced maximum in the mutual information," Phys. Rev. E, vol. 54, no. 3, pp. 2185-2188, 1996

[61] F. Chapeau-Blondeau, "Noise-enhanced capacity via stochastic resonance in an asymmetric binary channel," Phys. Rev. E, vol. 55, no. 2, pp. 2016-2019, 1997.

[62] I. Goychuk and P. Hänggi, "Stochastic resonance in ion channels characterized by information theory," Phys. Rev. E, vol. 61, no. 4, pp. 4272-4280, 2000.

[63] S. P. Strong, R. Koberle, R. R. de Ruyter van Steveninck, and W. Bialek, "Entropy and information in neural spike trains," Phys. Rev. Lett., vol. 80, no. 1, pp. 197-200, 1998.

[64] I. Goychuk and P. Hänggi, "Quantum stochastic resonance in parallel," New J. Phys., vol. 1, pp. 14.1-14.14, 1999

[65] L. B. Kish, G. P. Harmer, and D. Abbott, "Information transfer rate of neurons: Stochastic resonance of Shannon's information channel capacity," Fluct. Noise Lett., vol. 1, no. 1, pp. L13-L19, 2001

[66] A. Yakovleff and A. Moini, "From sensing to perceiving: An overview of the bugeye project," Proc. SPIE, vol. 2950, pp. 86-96, 1996. 
[67] D. Abbott, A. Bouzerdoum, and K. Eshraghian, "Future directions for motion detection based on the parallel computational intelligence of insects," in Proc. 23rd Euromicro Conf. New Frontiers of Information Technology, Budapest, Hungary, 1997, pp. 244-249.

[68] D. Abbott, A. Moini, A. Yakovleff, X. T. Nguyen, A. Blanksby, G. Kim, A. Bouzerdoum, R. E. Bogner, and K. Eshraghian, "A new VLSI smart sensor for collision avoidance inspired by insect vision," Proc. SPIE, vol. 2344, pp. 105-115, 1994.

[69] D. Abbott and A. Parfitt, "Extension of the vision paradigm to millimeter waves," Proc. SPIE, vol. 3207, pp. 103-106, 1997.

Gregory P. Harmer received the B.Sc. degree in applied mathematics and computer science in 1996 and the B.E. (Hons.1) degree in 1997 from the University of Adelaide, Australia, and the Ph.D. degree from the Department of Electrical and Electronic Engineering from the same university in 2001.

He now works at Sensor Research and Development, Maine.

Mr. Harmer was an invited speaker of UPoN'99, Adelaide.

Bruce R. Davis (M'78) received the B.E. (Hons.) degree in 1960, the B.Sc. degree in 1963, and the Ph.D. degree in 1969, all from the University of Adelaide, Australia.

He has been with the University of Adelaide since 1964 and is currently an Associate Professor of electrical and electronic engineering and Associate Dean (Postgraduate Matters) with the Division of Engineering and Mathematical Sciences. During 1970, he was with Bell Laboratories, Holmdel, NJ, studying various aspects of mobile radio communications and again in 1977, when he was involved in satellite systems research. In 1992, he was a vVisiting Scholar with the Communications Division of the Defence Science and Technology Organization (DSTO), Salisbury, South Australia, and was involved with high frequency data communication systems. His research interests are in mobile communication systems, satellite communications, and high frequency data communication systems. His most recent contribution is an innovative modification of importance sampling as applied to the simulation of digital satellite communications systems. He is co-authoring a text on noise for Cambridge University Press.

Dr. Davis is on the international scientific advisory committee of UPoN'99, held in Adelaide, Australia.
Derek Abbott (M'85-SM'99) received the B.Sc. (Hons.) degree in physics from Loughborough University of Technology, U.K., and the Ph.D. (distinction commended) degree in electrical and electronic engineering from Adelaide University, Australia.

He has led a number of research programs in the imaging arena, ranging from the optical to infrared to millimeter wave to terahertz (T-ray) regimes. From 1977 to 1986, he worked at the GEC Hirst Research Centre, London, U.K., in the area of visible and infrared image sensors. He has worked with nMOS, CMOS, SOS, CCD, GaAs, and vacuum microelectronic technologies and is now moving in the MEMS/nanotechnology arena. His expertise spans VLSI design, optoelectronics, device physics, noise, fabrication, and testing. Since emigrating to Australia, he has worked for Austek Microsystems, Technology Park, South Australia. Since 1987, he has been with Adelaide University, where he is presently an Associate Professor and the Director of the Centre for Biomedical Engineering (CBME). He is a founder member of the Centre for $\mathrm{GaAs}$ VLSI Technology (now CHiPTec), instituted in 1987, and served in an acting Deputy Director role (1989-1996) and was a Deputy Director (1996-1998). He has been consultant to various U.K. and Australian defense and industry organizations. He thas appeared on national and international television and radio and has also received scientific reportage in New Scientist, The Sciences, Scientific American, Nature, The New York Times, and Sciences et Avenir. He was the discoverer of the photovoltaic self-biasing internal-gain edge-effect within planar GaAs MESFETs and holds over 150 publications/patents. He has reviewed for IEEE TRANSACTIONSand is he co-authoring a text on noise for Cambridge University Press.

Dr. Abbott has been an invited speaker at over 50 institutions around the world, including Princeton, NJ; MIT, MA; Santa Fe Institute, NM; Los Alamos National Laboratories, NM; Cambridge, U.K.; Technion, Israel, and EPFL, Lausanne, Switzerland. He won the GEC Bursary (1977) and the Stephen Cole the Elder Prize (1998). He has served as an editor and/or guest editor for a number of journals including IEEE JOURNAL OF SOLID STATE CIRCUITS, CHAOS (AIP), Microelectronics (Elsevier), and Fluctuation Noise Letters (World Scientific). He has served on a number of IEEE and SPIE conference technical program committees, including the IEEE GaAs IC Symposium. 